GANIT J. Bangladesh Math. Soc. (ISSN 1606-3694) 38 (2017) 27-46

DOI: https://doi.org/10.3329/ganit.v38i0.39783

\title{
EXISTENCE OF PERIODIC TRAVELING WAVES IN THE KLAUSMEIER MODEL OF DESERTIFICATION AND ITS MODIFICATION: A COMPARATIVE STUDY
}

\author{
Md. A. S. Howlader*, Md. Ariful Islam Arif, L. S. Andallah,and M. Osman Gani \\ Department of Mathematics, Jahangirnagar University, Savar, Dhaka. \\ *Corresponding author, Email: salam_1971@yahoo.com
}

Received: 28-04-2018Ａccepted: 05-09-2018

\begin{abstract}
Self-organized and spatially periodic banded vegetation patterns have been observed in many semiarid ecosystems. In order to understand the mechanism of these patterns, we consider a system of reaction-advection-diffusion equations in a two-variable model of desertification. This work deals with the investigation of the existence of periodic traveling waves in a one-parameter family of solutions. In addition, we investigate the existence of periodic traveling waves as a function of water transport parameter in the model.
\end{abstract}

Keywords: Klausmeier model, periodic traveling waves, desertification, reaction-advectiondiffusion equations.

\section{Introduction}

In many landscapes around the world, the vegetation cover is sparse and exhibits spectacular organized spatial features[1] that can be either spatially periodic or random (namely labyrinthine, spotted or gapped). Commonly denoted as 'vegetation patterns' [2, 3]. Among these patterns, selforganized and spatially periodic banded vegetation patters are a striking characteristic feature of many semi-arid ecosystems [4,5]. Approximately 30\% of the emerged surface of the earth is covered with these patterns. Self-organized vegetation patterns have been observed to form on hillsides of the semi-arid regions with the limited availability of the water resources. These patterns are concentrated into bands, typically $100 \mathrm{~m}-250 \mathrm{~m}$ wide, running parallel to the hill's contours on gentle and uniform slopes of about $0.25 \%$ gradient. These stripes of vegetation are separated by gaps of essentially bare ground in semi-arid region, typically of width $200 \mathrm{~m}-1 \mathrm{~km}$, in which vegetation cover is almost absent or sparse $[3,4,6]$. The wavelength of this pattern is typically about $1 \mathrm{~km}$ for shrubs and trees, but the shorter range is observed for grasses [4, 5]. In this paper, the stripes of vegetation on a uniform hillslope will be in the center of attention.

Most of the authors [7-9] agreed that the underlying cause of vegetation patterning is the competition for water and the positive feedback between water availability and plant growth. The verbal explanation for the mechanism indispensable for the maintenance and movement of vegetation bands is as follows: Rain falling on the bare grounds between bands/stripes of 
vegetation doesn't infiltrate into the soil, but it flows downhill to the next vegetation band where it soaks in and enables the maintenance of the vegetation band. This water is then exhausted upon reaching the downslope edge of the stripe, which causes again a bare ground. The moist soil on the uphill edge of the band creates a tendency to be migrated on the uphill edge. This also explains a most striking feature of this pattern formation such that the stripes slowly move uphill as time passes. This is because of the fact that vegetation can grow and survive at the upper area of a vegetation stripe, because there is enough moisture. On the other hand, the vegetation dies at the lower area of a vegetation stripe, because it is not moist enough[10].However, the details of the banded vegetation process remain controversial for limited long-term data.

It is to mention thatarid and semi-arid landscapes cover about one-third of the Earth's surface. Many deserts are expanding due to drought. So that self-organized vegetation patterns are an important indication in understanding the potential early warning signals of environmental change and imminent regime shifts. Therefore, the aim of studying this particular phenomenon is therefore to be able to give early warning signals in regions which are directly threatened by desertification. Therefore, they have been the subject of intensive research over the last decade. Field experiments are not cost-effective and not so easy to carry out, because of geographical remoteness and physical harshness in most instances of the potential study sites of banded vegetation. Moreover, there are no laboratory replicates of this phenomenon, and in most cases onehas to rely onusing remote sensing satellite images. However, the time scale of pattern evolution is very slow (it may take more than one decade), and such observational data are ineffective to investigate the implications of changes in environmental parameters such as rainfall levels. Although it has obvious limitations, this relevant information has helped us to predict how vegetation pattern change in environmental shifts. Being of limited utility given the long space and time scales involved in the vegetation pattern formation process, theoretical models have emerged as an important research tool for studying these patterns, and a range of mathematical models have been developed [11].

The first continuum mathematical model for vegetation patterning is the Klausmeier model[10], and this model and most of its extension [7,12-15] has broadly been used as an important tool for researcher and have been studied broadly in both computer-based simulation [16,17], and analytical studies [6,18-20]. This model is an important tool to understand the mechanisms of striped vegetation, and a simplification for using the single variable of water.

It is mentioned earlier that, in many semi-arid landscapes vegetation is often self-organized into large-scale spatial patterns. The natural phenomenon of these patterns is particularly banded and periodic, this motivates us to study the existence of periodic traveling wave solutions (PTWs) in one dimension. Therefore, in this paper, we study the existence of the PTWs numerically by using a two-component reaction-advection-diffusion system in one-parameter family of solutions in one dimension. The focus of thepresent study is to investigate the existence of PTWs of the modified 
Klausmeier model to understand the mechanism of banded vegetation patterns in semi-arid ecosystem as introduced by van der Stelt et al. [20].

The rest of this paper is arranged as follows: In section 2 and 3, we briefly describe the Klausmeier model and modified Klausmeier model of desertification respectively. Section 4illustrates the methods of computation. In section 5, we present our results in one dimension, for various parameters with discussion. Finally,section 6 is the conclusion. This section underlies the significant results of the present study, and gives further directions for future research on prospective areas.

\section{A brief outline of the Klausmeier model}

The original Klausmeiermodel, when appropriately non-dimensionalized [6,10] consists of two partial differential equations mentioning the plant biomass density and water density and is given as follows:

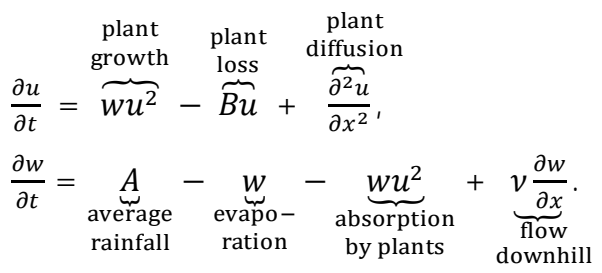

This model is a dynamical system of interaction between plant and water to get physical vegetation pattern. The reaction terms $f(u, w)=w u^{2}-B u$ and $g(u, w)=A-w-w u^{2}$ describe the local kinetics of variables $u$ and $w$. Here, $u(x, t)$ and $w(x, t)$ represent plant biomass density in space $x$, measured in the uphill direction (positive ' $x$ ' direction) at a constant migration speed, and water density respectively at time ' $t$ '. It is a need to mention that this model is deliberately simple and conceptual, because it does not separate water into surface and sub-surface components. The model assumes constant rainfall, with water lost via both evaporation and absorption by plants. The assumption of proportionality between evaporation and water density is consistent with both more detailed modeling and field data [21].Per capita plant growth is assumed to be jointly proportional to water density and vegetation biomass. The dependence on biomass reflects the positive correlation between the infiltration capacity of soil and its vegetation level in semi-arid environments [4,22,23]. The non-linear term $w u^{2}$ in $(1 a)$ denotes plant growth, which is considered to be proportional to water availability and in $(1 b)$ it is regarded as the uptake feedback of water in order to promote the plant growth [10].Plant loss is assumed to occur at a constant per capita rate, which may include herbivory. Finally, water flows downhill while plant dispersal is modeled by linear diffusion. The advection term $v \frac{\partial w}{\partial x}$ in $(1 b)$ means that spatial patterns are not stationary; rather they move in the positive $x$ direction (uphill) at a constant rate. There has been a long-running debate in the ecological literature about this uphill migration, with some field studies reporting stationary patterns [24]. 
The model contains three parameters, $A, B$ and $v$ which are dimensionless combination of ecological quantities. The parameter $A$ is the control parameter as well as bifurcation parameter, represents precipitation as a source term. However, the parameter $A$ is proportional to mean annual rainfall. Although, in most semi-arid regions rainfall typically occurs at a certain periods of the year and sometimes in relatively brief storms [25]. The parameter $B$ measures plant losses including both natural death and the effect of any herbivory, and $v$ is the slope gradient (steepness of the slope), which controls the rate at which water flows downhill. The typical values of these ecological parameters estimated by Klausmeier (1999) are $B=0.45$ for grass, $B=0.045$ for trees; in either case $v=182.5$ for a typical slope with vegetation stripes and we choose those parameter values compatible for grass.

\section{Modified Klausmeier model of vegetation}

In this study, we also consider a modified version of the Klausmeier [10] model (see Equation (1)) by appending an additional term water diffusion which is also reported in [26-28].The modified version of the basic Klausmeier model is a reaction- advection-diffusion system of PDEs in which the spatial patterns of vegetation is the result of competition for water. The suitably nondimensionalized form of the model equations [6,10,17] consist of two state variables $u$ and $w$ describing the plant biomass density and water density respectively. They are functions of both spatial variable ' $x$ ' $(x \in \mathbb{R})$, measured in the uphill direction and the temporal variable ' $t$ '. It is to be mentioned that we will consider a gentle slope and behavior in one spatial dimension ' $x$ ', following the description in [20,29], which is sufficient for banded patterns. The model is given by (2) as follows:

$$
\begin{aligned}
& \frac{\partial u}{\partial t}=\overbrace{w u^{2}}^{\begin{array}{c}
\text { plant } \\
\text { growth }
\end{array}}-\overbrace{B u}^{\begin{array}{c}
\text { plant } \\
\text { loss }
\end{array}}+\overbrace{d_{u} \frac{\partial^{2} u}{\partial x^{2}}}^{\begin{array}{c}
\text { plant } \\
\text { disparsal }
\end{array}} \\
& \frac{\partial w}{\partial t}=\underbrace{A}_{\begin{array}{c}
\text { average } \\
\text { rainfall }
\end{array}}-\underbrace{w}_{\begin{array}{c}
\text { evap- } \\
\text { oration }
\end{array}}-\underbrace{w u^{2}}_{\begin{array}{c}
\text { absorption } \\
\text { by plants }
\end{array}}+\underbrace{v \frac{\partial w}{\partial x}}_{\begin{array}{c}
\text { flow } \\
\text { downhill }
\end{array}}+\underbrace{d_{w} \frac{\partial^{2} w}{\partial x^{2}}}_{\begin{array}{c}
\text { diffusion } \\
\text { of water }
\end{array}} .
\end{aligned}
$$

Klausmeier, in his original model, included an advection term for water instead of water diffusion on the phenomenological ground. Adding water diffusion in the Klausmeier model is valid due to following reasons:

(a) Because of the physical ground of diffusion term it can be derived from shallow water equations [30]. However, Ursino[31] showed that a diffusion term always accompanies the advection term when water transport is derived from the Richards equation for soil water flow.

(b) This term admits capturing the movement of rainwater by the influence of spatial differences in the rate of infiltration [12]. 
A key component of (2) is the non-linear term $w u^{2}$, assumed to be proportional to water absorption (uptake) by plants and this term reflects the fact that the presence of root networks and the higher levels of organic matter in the soil increases the infiltration of rainwater into the soil $[22,23]$ to promote the plant growth. The parameter $A$ is the control parameter as well as bifurcation parameter, represents the rate of mean annual rainfall (as a source term), which typically occurs in discrete storm events in semi-arid regions [25,28], and $-w$ represents evaporation as a loss term. The dimensionless parameter $B$ measures plants losses including both natural death and the effect of any herbivory. The term $B u$ describes plant biomass which is considered to have a simple linear form. The diffusion term $d_{u} \frac{\partial^{2} u}{\partial x^{2}}$ represents the spread of plants by both clonal reproduction and seed dispersal. The parameter $d_{u}$ indicates the plant dispersal coefficient. The dimensionless quantity $v$ represents the slope gradient (steepness of the slope), which controls the rate at which water flows downhill. The advection term $v \frac{\partial w}{\partial x}$ represents the surface runoff, which assumed to be proportional to $w$. This term was derived from shallow water theory representing the downhill flow of water [30]. It isa need to mention that the water diffusion parameter $d_{w}$ was first introduced by Kealy and Wollkind [19]. Recall that, this diffusion term has a physical meaning, since it can be obtained from both the Richards equation for soil water flow [31], and the shallow water equations [30]. However, the values of the water diffusion coefficient $d_{w}$ in $(5.1 b)$ was not estimated by those authors. Ursino[31] estimated the values of the parameter $d_{w} \in(7.5,110)$. Comparing the rainfall range giving patterns, Siteur et al.[28] used the larger value of $d_{w}=500$ according to field data.However the suitable value of $d_{w}$ still remains unclear. Intuitively, homogeneous vegetation state arise by sufficiently high rainfall, while a complete desert state is the result of very low rainfall. Again, intermediaterainfall levels are very low that it can't maintain homogeneous vegetation cover, butare compatible with vegetation stripes. As we are intended to restrict our attention in the response of the system to changes in rainfall, we consider rainfall $A$ as a free parameter as well as bifurcation parameter and choose $A \in[0.1,3.5]$. Here, diffusion coefficient $d_{w}=500$, will typically be much larger than that of plant dispersal coefficient of $d_{u}=1$, this additional term $d_{w}$ expedites the pattern forming potential of the model, because water diffusion intuitively improves flow from bare ground to vegetated patches. Equation (2) is considered as one of the simplest and earliest models dedicating vegetation pattern formation in semi-arid ecosystems, and remains gigantic in use [19,28,29,31]. Moreover, it falls in the broader class of reaction(-advection-)-diffusion models referred to as activator-depleted substrate systems [32] in which vegetation acts as an activator and rainwater acts as a substrate.

\section{Methods of computation}

Vegetation stripes that slowly migrate in the uphill direction mean that they are periodic travelling waves, can be studied via the ansatz:

$$
u(x, t)=U(z), w(x, t)=W(z),
$$


where a travelling wave coordinate $z=x-c t, x$ and $t$ are the one dimensional space and time coordinate respectively, and $c$ is the pattern migration speed. By using these solution forms, we obtain the following equations form:

Similarly,

$$
\begin{aligned}
& \frac{\partial u}{\partial t}=\frac{d U}{d z} \frac{\partial z}{\partial t}=-c \frac{d U}{d z}, \\
& \frac{\partial u}{\partial x}=\frac{d U}{d z} \frac{\partial z}{\partial x}=\frac{d U}{d z}, \\
& \frac{\partial^{2} u}{\partial x^{2}}=\frac{d^{2} U}{d z^{2}} .
\end{aligned}
$$

$$
\begin{aligned}
& \frac{\partial w}{\partial t}=\frac{d W}{d z} \frac{\partial z}{\partial t}=-c \frac{d W}{d z}, \\
& \frac{\partial w}{\partial x}=\frac{d W}{d z} \frac{\partial z}{\partial x}=\frac{d W}{d z}, \\
& \frac{\partial^{2} w}{\partial x^{2}}=\frac{d^{2} W}{d z^{2}} .
\end{aligned}
$$

Substituting the above results into the modified Klausmeier model (2), we obtain the following second order ODEs

$$
\begin{aligned}
& d_{u} \frac{d^{2} U}{d z^{2}}+c \frac{d U}{d z}+W U^{2}-B U=0, \\
& d_{w} \frac{d^{2} W}{d z^{2}}+(c+v) \frac{d W}{d z}+A-W-W U^{2}=0 .
\end{aligned}
$$

These ordinary differential equations must be reduced to a first order four-dimensional traveling wave ODEs as follows:

$$
\begin{aligned}
& \frac{d U}{d z}=P, \\
& \frac{d P}{d z}=\frac{d^{2} U}{d z^{2}}=\frac{1}{d_{u}}\left(-c P+B U-W U^{2}\right), \\
& \frac{d W}{d z}=Q, \\
& \frac{d Q}{d z}=\frac{d^{2} W}{d z^{2}}=\frac{1}{d_{w}}\left[-(c+v) Q-A+W+W U^{2}\right] .
\end{aligned}
$$

A periodic traveling wave solution is a periodic orbit or a limit cycle solution of this ODE system (4). In simple cases, the branch of this solution is monotonic in the parameters, and has at least one end terminating at a Hopf bifurcation point [33]. However, in some complex problems the branch begins and ends at homoclinic solution. In that case, the user is required to provide a periodic traveling wave solution as an initial starting solution from the simulation of the partial differential equation for a pair of control parameter and wave speed values for the continuation.

\subsection{Homogeneous steady-state solutions}

Periodic travelling waves typically develop from a Hopf bifurcation of a steady state solution of the travelling wave equations. The first step in investigating the pattern-forming potential of the 
model (2) is to determine the spatially homogeneous steady states.Setting the L.H.S of the first order ODEs (4) to zero provides a system of algebraic equations that can easily be solved for the unique steady-state with $U$ and $W$ both non-zero:

$$
\begin{aligned}
& P=0, \\
& B U-W U^{2}=0, \\
& Q=0, \\
& -A+W+W U^{2}=0 . \\
& (5) \Rightarrow \quad U(B-W U)=0 .
\end{aligned}
$$

For all parameter values, (2), has a stable trivial steady state:

if $U=0$, then $W=A$, from (6), corresponding to bare ground, without vegetation.Again, if $(B-W U)=0$, then

$$
U=B / W .
$$

$(5)+(6) \Rightarrow W=A-B U \Rightarrow W=\left(A \pm \sqrt{A^{2}-4 B^{2}}\right) / 2$, by using (7)

$\therefore U=2 B /\left(A \pm \sqrt{A^{2}-4 B^{2}}\right),[$ from $(7)]$.

Here, we observe that, when $A \geq 2 B$, there are also two other homogeneous steady states which arise from a saddle-node bifurcation

$$
\begin{aligned}
& U=U_{1} \equiv \frac{2 B}{A+\sqrt{A^{2}-4 B^{2}}}, W=W_{1} \equiv \frac{A+\sqrt{A^{2}-4 B^{2}}}{2}, \\
& \text { and } \quad U=U_{2} \equiv \frac{2 B}{A-\sqrt{A^{2}-4 B^{2}}}, \quad W=W_{2} \equiv \frac{A-\sqrt{A^{2}-4 B^{2}}}{2} .
\end{aligned}
$$

The first of thse (8) is always unstable to homogeneous peturbations; the second is the key equilibrium from which patterns develop. This steady state is linearly stable to homogeneous perturbations whenever $B<2$. For larger values of $B$ and small $A$ (9) can become unstable, giving complecated local dynamics including a limit cycle, but realistic parameter values for plant growth in semi-arid environments imply $B<2$.

\section{Numerical results and discussion}

Figure 1 shows the existence of PTW solutions of (1) as a function of the rainfall parameter $A$ and the wave speed or the pattern migration speed $c$. Here we consider the parameter $A$ as rainfall parameter as well as bifurcation parameter in our study while the other parameters are $B=$ 0.45 and $v=182.5$. 


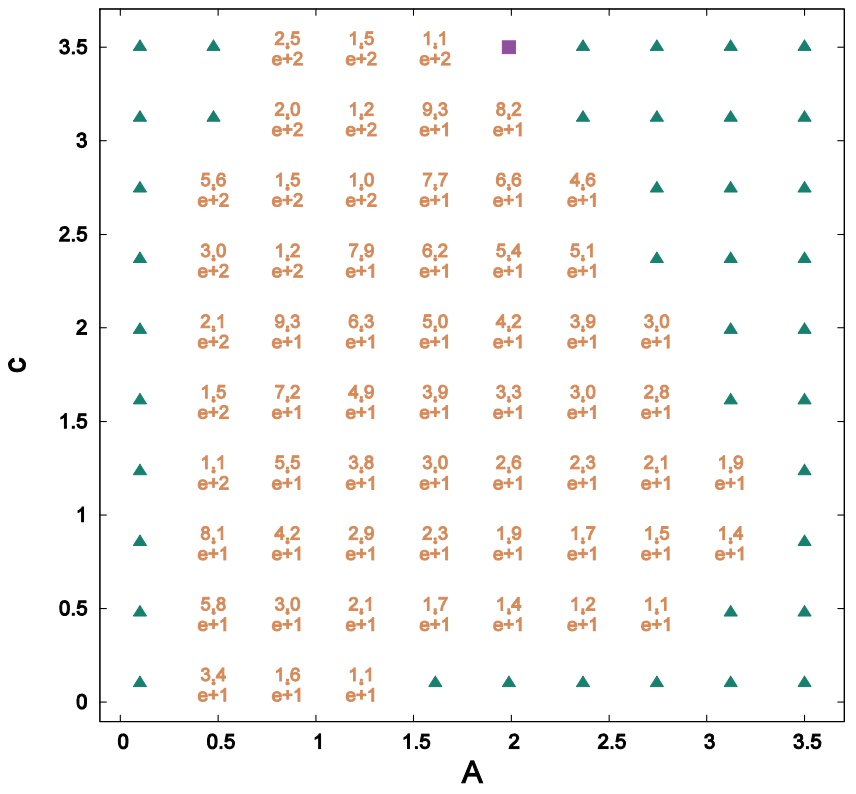

Fig. 1: The existence of periodic traveling wave solutions of (1) as a function of the parameter $A$ and the wave speed $c$.

The parameter $A$ lies between 0.1 and 3.5 i.e., the range $0.1 \leq A \leq 3.5$, for $A$ has been chosen. The symbols and values exhibit the results carried out over a domain of $10 \times 10$ cells in the $A-c$ parameter plane: a filled triangle indicates that there is no PTW existsat that point; a filled square indicates that no PTW exists there but solution is convergent at that point. The values on the parameter plane signify the periods of the PTW solutions of a given point of the control parameter $A$ and the wave speed $c$. The periodic traveling wave also exists for other values of the parameter $A$, outside of the above range. However, we are only interested to understand the periodic pattern solutions for the model (1) within the above range. The Figure was calculated and plotted using the software package WAVETRAIN [33].

Figure 2 indicates two periodic traveling wave solutions of (1) as a function of the control parameter $A$ and the wave speed $c$.In parts $(a)$ and $(b)$, symbols $U, V$, and $W$ are the internal name of state variables of (1). (a) The first one is for the parameter $A=0.5$ and the wave speed $c=1.3$. The period of the wave is $=108.0996$. (b) The second one is for the parameter $A=2.7$ and the wave speed $c=1.3$. In this case the period of the wave is $=21.9949$. The other parameter values are $B=0.45$ and $v=182.5$.These Figures indicate that, increasing the value of control parameter $A$ decreases the periods of the PTW solutions at constant wave speed. However, the periods are not changing quickly as $A$ changes. The other parameter values are the same as in Figure 1. 


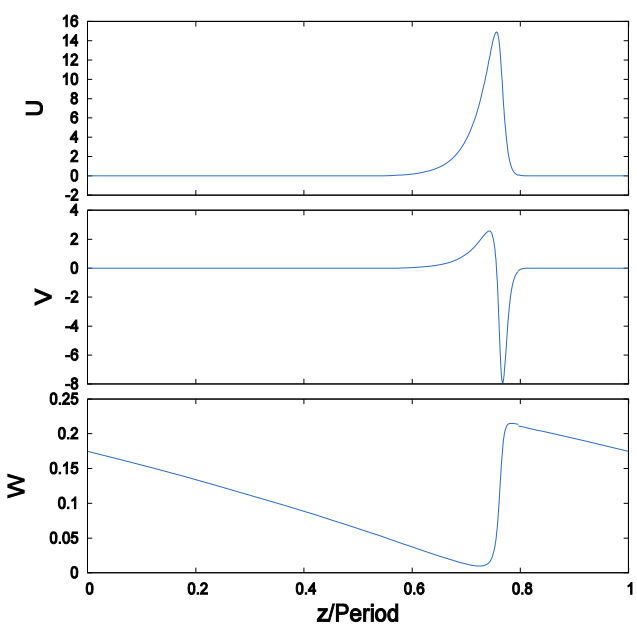

(a)

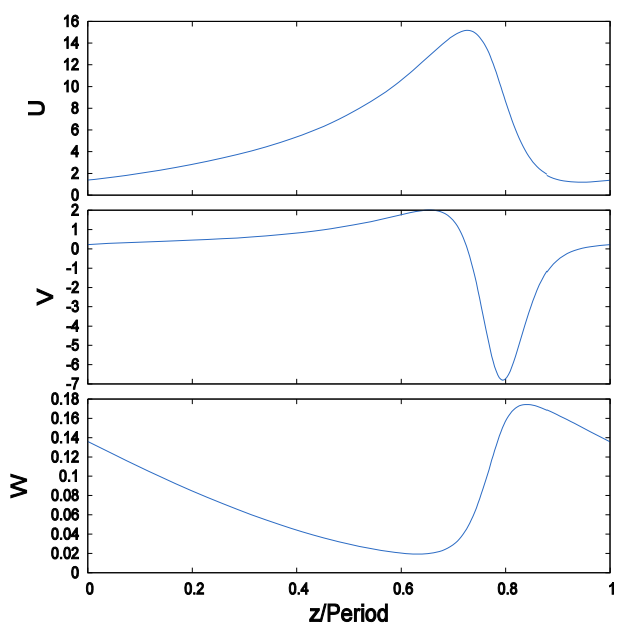

(b)

Fig. 2: Two periodic traveling wave solutions of (1) as a function of the parameter $A$ and the wave speed $c$.

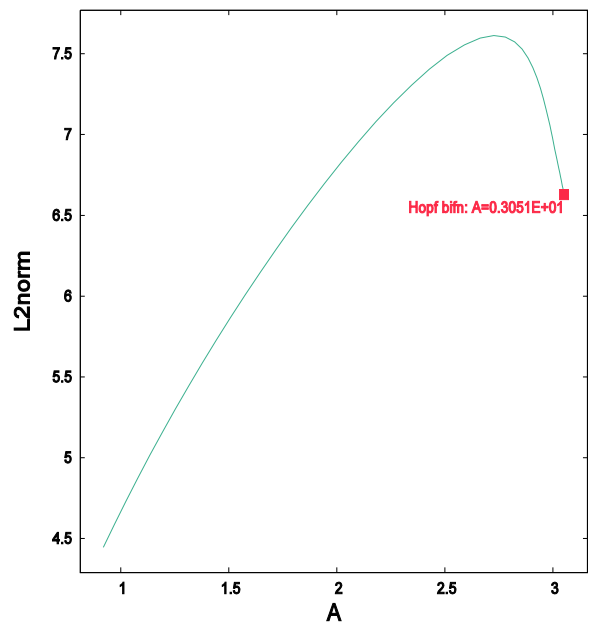

(a)

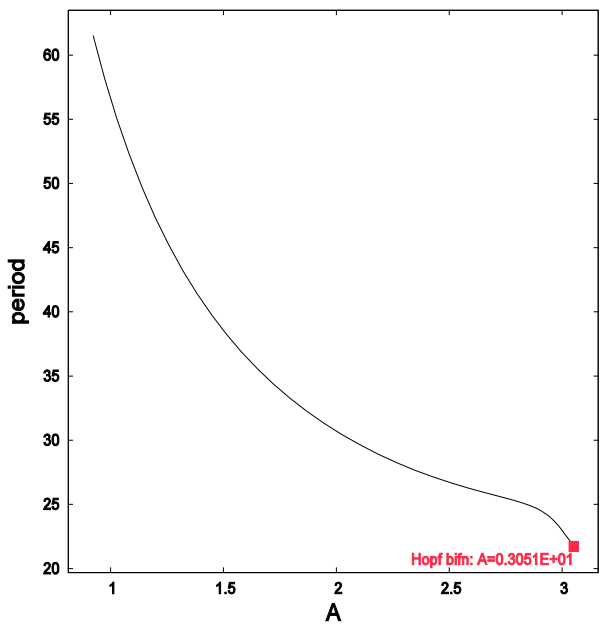

(b)

Fig. 3: The branches of periodic traveling wave solutions of (1) with wave speed $c=1.5$.

Figure 3 exhibits the branches of periodic traveling wave solutions or the bifurcation diagrams of (1) with fixed wave speed $c=1.5$.The other parameter values are the same as in Figure 1.The curves were calculated and plotted using the software package WAVETRAIN [33].Figure 3( $a$ ) illustrates the $L 2$-norm of the solution of the traveling wave ODEs of (1), plotted as a function of the rainfall parameter $A$. In Figure 3 $(b)$, pattern wave period is plotted as a function of the rainfall parameter $A$. The branch of solution in each plot $(a)$ and $(b)$ initiates from a Hopf bifurcation point 
marked by filled red square at $A=3.051$ and terminates at homoclinic solution by decreasing values of $A$. When the value of the parameter $A$ is increased, the corresponding period decreases (Fig. 3(b)) and L2-norm (Fig. 3(a)) of the solution increases. It is well known that the existence of periodic traveling wave solutions depend on the parameter values of the PDE system (1) and also on the wave speed $c$. If PTW solutions exist for a parameter setting of the PDEs, then they exist for a range of wave speed $c$ [34].

\subsection{Existence of PTWs as a function of water diffusion, $d_{w}$}

We consider the effect of both diffusion and advection terms for water in (2). Therefore, we add an additional termwater diffusion $d_{w} \frac{\partial^{2} w}{\partial x^{2}}$ to the right side of $(2 b)$.Again, we want to limit our study to one-space dimension. We investigate the existence of periodic pattern solutions or PTWs in the $A-c$ parameter plane by varying the values of water diffusion coefficient $d_{w}$. Since for $d_{w}=0$, the change of solutions for $d_{w}$ is not dramatic. Therefore, in this section, we consider the results for $d_{w}=0.01$ is similar to the Klausmeier model (1).The other parameter values remain constant as in Table 1. Figure 4(a-d) illustrates the region of the $A-c$ parameter plane in which pattern exists as a function of the water diffusion parameter, calculated via the WAVETRAIN [33,35].A numerically determined periodic orbit from the PDEs is used as the beginning point for the continuation. Here, we consider the rainfall parameter $A$ as a free parameter from an ecological point of view, and the range of the control parameter $A$ in the rainfall-wave speed parameter plane is $0.1 \leq A \leq 3.5$.The triangles and values on the parameter plane exhibitthe results over a domain of $10 \times 10$ grids in the parameter plane. The triangle indicates that there is no PTW at that point, and the value on the parameter plane indicates the period of the PTW solution of a given point of the parameter $A$ and the wave speed $c$.The orange line (right boundary) denotes the locus of Hopf bifurcation points in the traveling wave ODEs(4).The pink line (left boundary) indicates the locus of homoclinic solutions, where the period (wavelength) is infinite. The homoclinic solution is actually approximated by a locus of the PTWs with large finite period 3000. Therefore, the pattern forming region in the parameter plane is bounded right by a locus of Hopf bifurcation points, and bounded left by a locus of homoclinic solutions. We observe that the locus of homoclinic solutions and the locus of Hopf bifurcation points slightly change due to the increasing values of the water diffusion parameter $d_{w}=0.01,100,300$ and 500 respectively (see Fig. 4). In Figure 4, if the values of $d_{w}$ increase from 0.01 to 500 , the PTWs region gets shrink gradually near the region having large wave speedas the water diffusion increases from panel (a) to panel (d).

Table 1: Typical set of parameter values in (2) used in the numerical computation.

\begin{tabular}{|l|c|c|c|c|c|}
\hline Parameter & $A$ & $B$ & $v$ & $d_{u}$ & $d_{w}$ \\
\hline Value & varied & 0.45 & 182.5 & 1 & varied \\
\hline
\end{tabular}




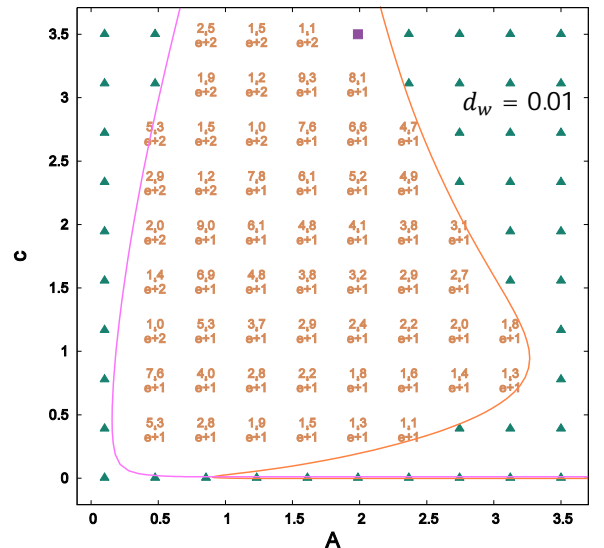

(a)

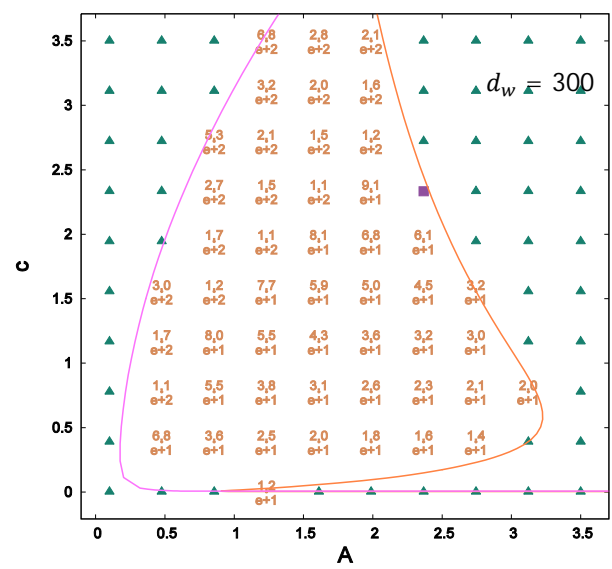

(c)

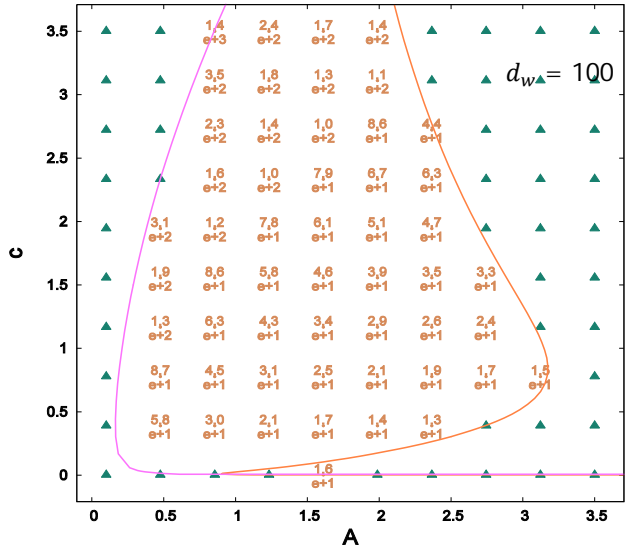

(b)

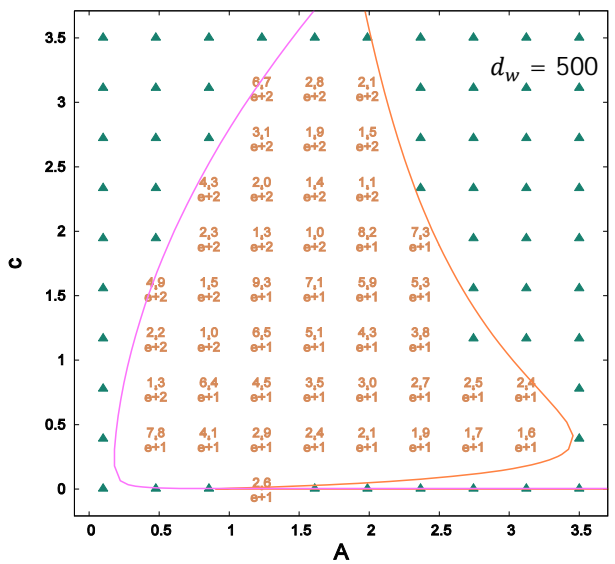

$(d)$

Fig. 4: An illustration of the part of the $A-c$ parameter plane giving the existence of PTWs of model (2) for (a) $d_{w}=0.01,(b) d_{w}=100,(c) d_{w}=300$ and $(d) d_{w}=500$ as a function of the rainfall parameter $A$ and the pattern migration speed $c$.

\subsection{Bifurcation diagrams of PTWs with fixed rainfall as a function of water diffusion, $d_{w}$}

Figure 5 illustrates the bifurcation diagrams of the PTWs in the model (2) for the increasing water diffusion parameter $(a) d_{w}=0.01,(b) d_{w}=100,(c) d_{w}=300$ and $(d) d_{w}=500$ by fixing the rainfall parameter at $A=2.5$ with other parameter values remaining the same as in Table 1.The dispersion curves $[36,37,38]$ demonstrate that the PTW branches begin from a Hopf bifurcation point marked by filled red square in each panel at $c=0.3366,0.3044,0.1726$, and 0.0878 and end at Hopf bifurcation point at $c=2.622,2.398,2.079$, and 1.890 respectively from panel to panel (see Figs. 5( $a-d)$ ). 

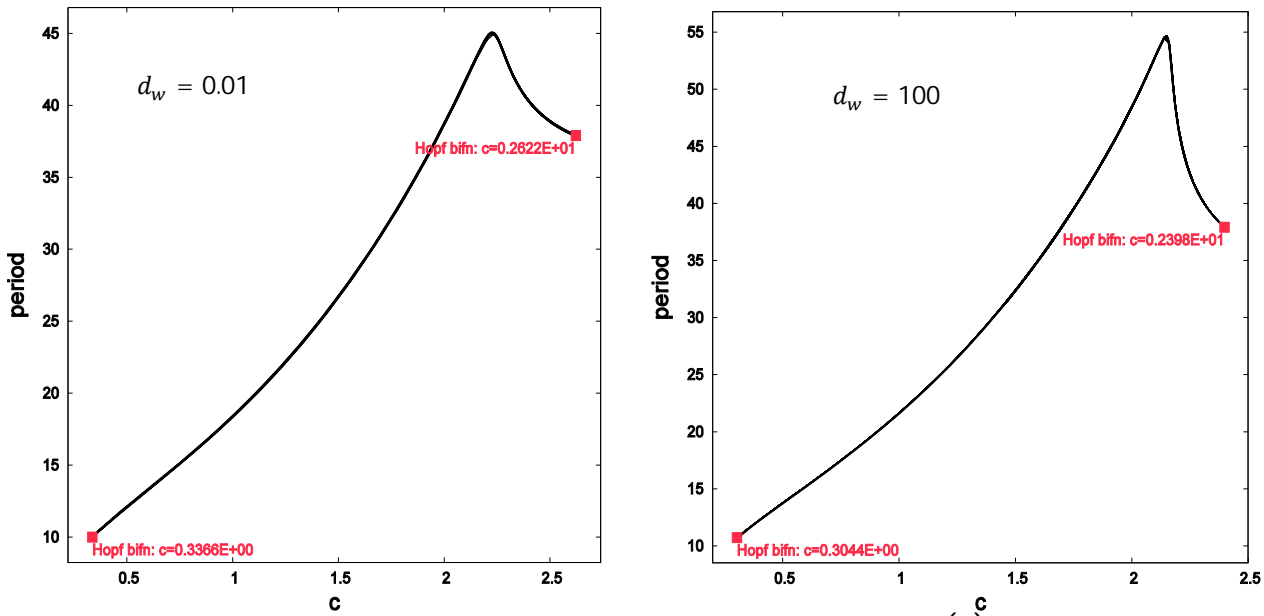

(a)

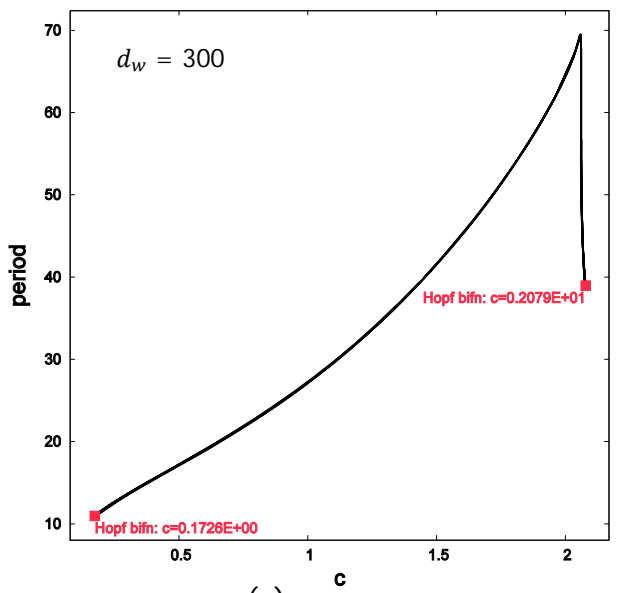

(c)

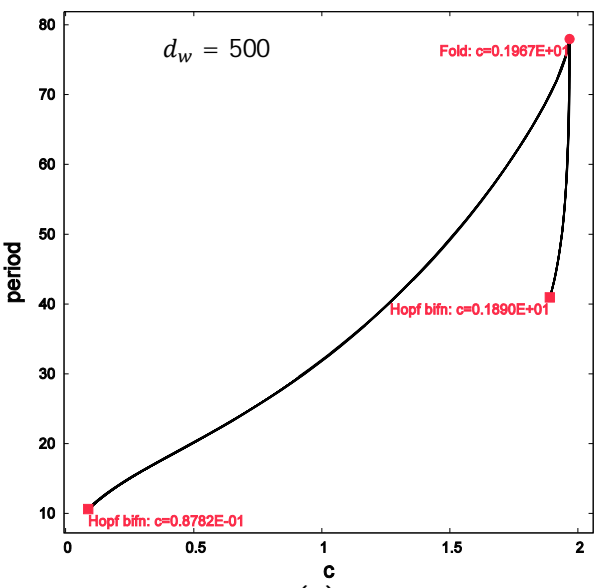

(d)

Fig. 5: The different bifurcation diagrams (dispersion curves) of the PTWs in model (2), plotted wave period as a function of the wave speed $c$ for fixed $A=2.5$. The water diffusion coefficients for the panels are at $(a)$ $d_{w}=0.01,(b) d_{w}=100,(c) d_{w}=300$ and $(d) d_{w}=500$.

Therefore, we deduce that the range of the wave speed between Hopf bifurcation points decreases from panel to panel for the increasing values of water diffusion $d_{w}$. We observe that the spatial wave period is increasing with respect to the wave speed $c$ until the period reaches its maximum value and eventually decreases. It is seen that there exists a fold bifurcation point marked by filled red circle at $c=1.967$ in the Figure $5(d)$.

\subsection{Bifurcation diagrams of the PTWs: Wversus $A$}

Figure 6 shows the different branches of the PTWs of (2), plotted W as a function of the rainfall parameter $A$, by fixing the wave speed at $c=1.5$. The water diffusion coefficients for the panels are at $(a) d_{w}=0.01,(b) d_{w}=100,(c) d_{w}=300$ and $(d) d_{w}=500$. The other parameter values are 
$d_{u}=1, B=0.45$, and $v=182.5$. Legend on the top of each panel shows the color of the locus of each solution branch. Note that the same line color is used for the $\mathrm{W}_{\max }$ and $\mathrm{W}_{\min }$. The light green line(middle line) in each panelindicates the L2-norm of the solution of the traveling wave ODEs (4). The blue lines (upper line and lower line) indicate the loci of the maximum and minimum of the water density variable $\mathrm{W}$ respectively.

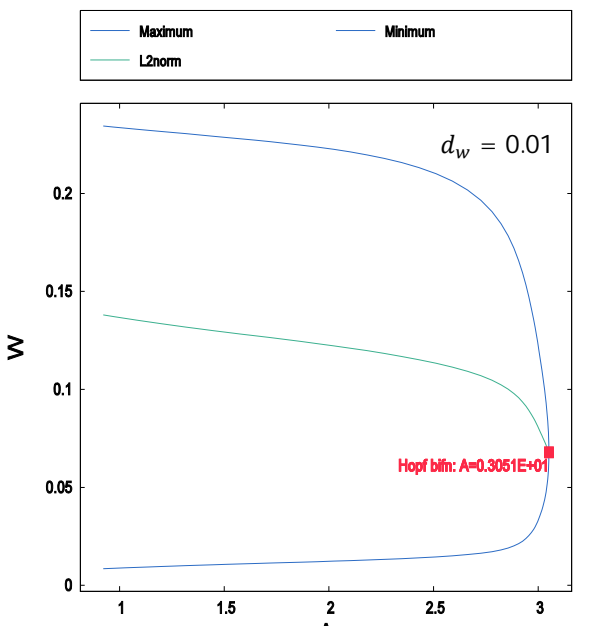

(a) ${ }^{\mathrm{A}}$
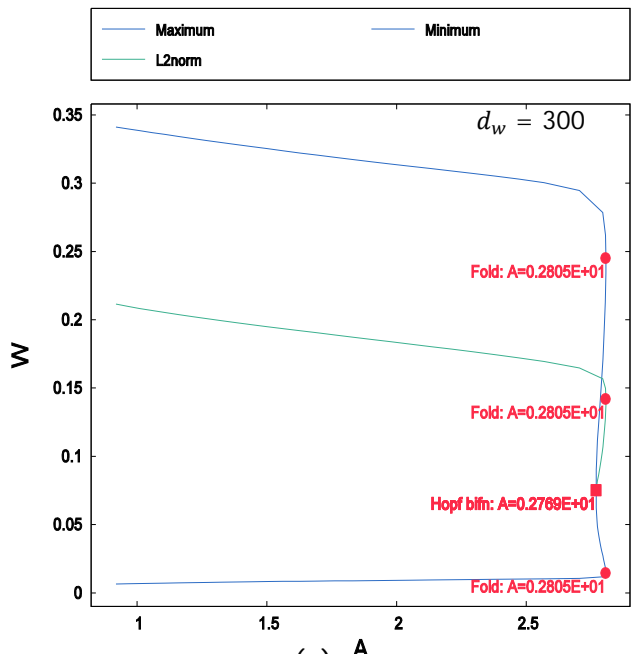

(c) $\mathrm{A}$
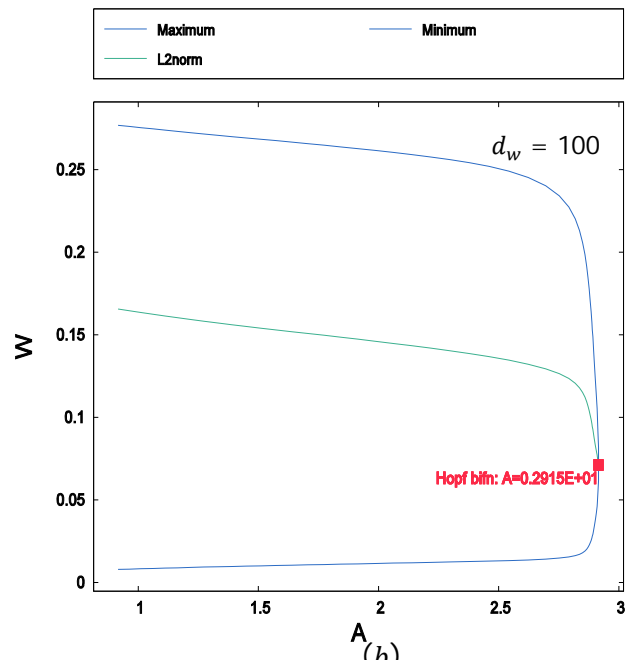

(b)
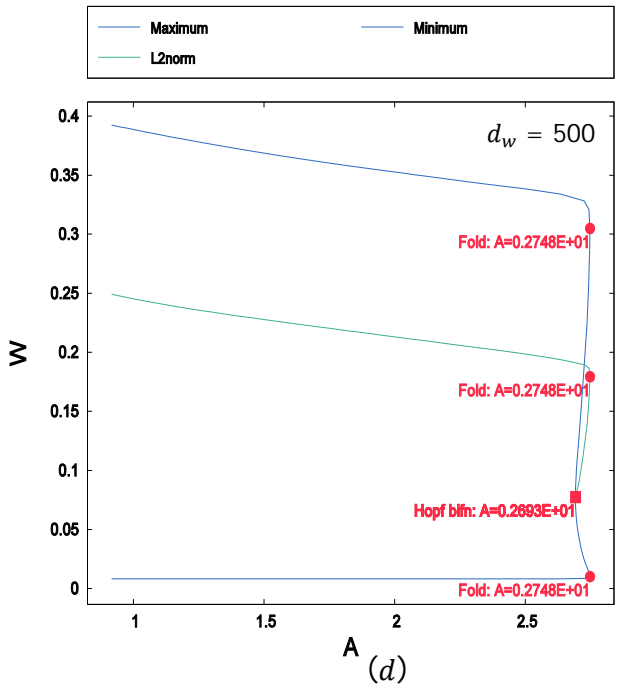

Fig. 6: The different bifurcation diagrams of the PTWs in the model (2), plotted $\mathrm{W}$ as a function of the rainfall parameter $A$ for fixed migration speed $c=1.5$.

The branches of solution begin from a Hopf bifurcation point marked by filled red square at $A=$ $3.051,2.915,2.769,2.693$ respectively for $d_{w}=0.01,100,300$, and 500. We observe that the values of the Hopf bifurcation points decrease gradually for the increasing values of $d_{w}=0.01$, 100,300 , and 500 respectively. In other words, increasing the water diffusion decreases the value 
of the rainfall parameter in order to born of the PTWs. There exists a fold point on the L2-norm marked by filled red circle at $A=2.805,2.748$ respectively for $d_{w}=300$, and 500. There exist also two fold points marked by filled red circles at $A=2.805,2.748$ on the locus of maximum and minimum water density variable $\mathrm{W}$ respectively for $d_{w}=300$, and 500 . We also observe that there exists no fold point for $d_{w}=0.01$, and 100. It is to be mentioned that the $\mathrm{W}_{\max }$ increases as a function of $d_{w}$, e.g., for $A=1.0$, theW $\mathrm{W}_{\max } \approx 0.25,0.29,0.34,0.38$ respectively when $d_{w}=$ $0.01,100,300$, and 500. But, the $\mathrm{W}_{\min }$ remains almost constant for any value of $A$.

\subsection{Bifurcation diagrams of the PTWs: Wversus c}

Figure 7 shows the different branches of the PTWs of (2), plotted W as a function of the wave speed $c$, by fixing the rainfall parameter at $A=2.5$. The water diffusion coefficients for the panels are at $(a) d_{w}=0.01,(b) d_{w}=100,(c) d_{w}=300$ and $(d) d_{w}=500$. The values of the other parameter are the same as in Figure 6. Legend on the top of each panel shows the color of the locus of each solution branch. The same line color is used for the $\mathrm{W}_{\max }$ and $\mathrm{W}_{\min }$. The light green line (middle line) in each panel exhibits the $L 2$-norm of the solution of the traveling wave ODEs (4). The locus of the maximum and minimum of the water density variable $\mathrm{W}$ is indicated by the blue line (upper line and lower line) from panel $(a)$ to panel $(d)$. The branches of solution initiate from a Hopf bifurcation point marked by filled red square at $c=0.3366,0.3044,0.1726,0.0878$ respectively for $d_{w}=0.01,100,300$, and 500. And, the branches of solution terminate at a Hopf bifurcation point at $c=2.622,2.398,2.079$, and 1.890 respectively for $d_{w}=0.01,100,300$, and 500.The branches of PTWs are bounded with $c \in[0.3366,2.622]$ for $d_{w}=0.01, c \in[0.3044,2.398]$ for $d_{w}=100, c \in[0.1726,2.079]$ for $d_{w}=300$ and $c \in[0.078,1.890]$ for $d_{w}=500$. It is to be mentioned that the ranges of the wave speed $c$ between the Hopf bifurcation points decrease gradually for the increasing values of $d_{w}=0.01,100,300$, and 500 respectively.
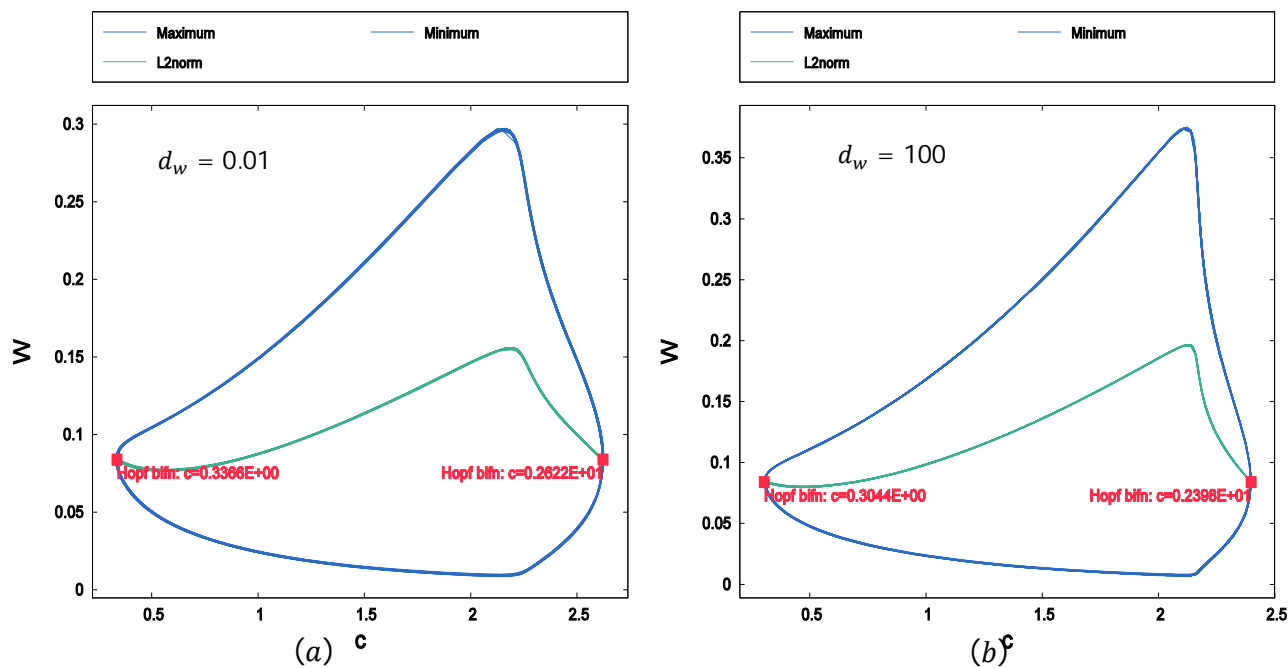


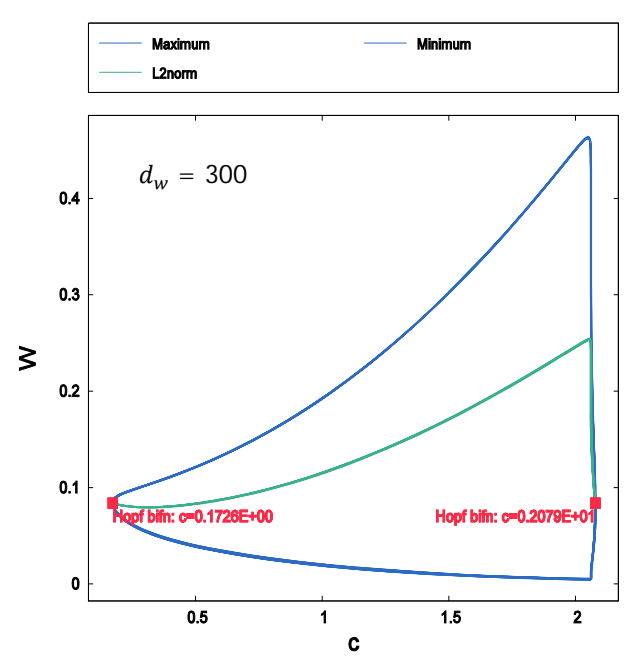

(c)

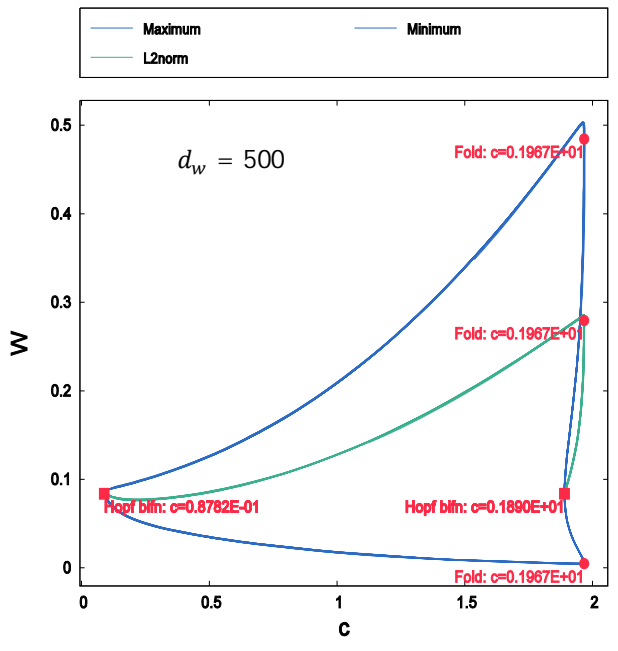

(d)

Fig. 7: The different bifurcation diagrams of the PTWs in the model (6.1),plotted W as a function of the migration speed $c$ for fixed rainfall parameter $A=2.5$.

There exists a fold point or a saddle-node bifurcation point marked by filled red circle on the L2norm at $c=1.967$ when $d_{w}=500$. There alsoexist two fold points marked by filled red circles at $c=1.967$ on the locus of maximum and minimum water density variable $\mathrm{W}$ for $d_{w}=500$. We observe that there exists no fold point for $d_{w}=0.01,100$, and 300 .

\subsection{Bifurcation diagrams of the PTWs: U versus $A$}

Figure 8 illustrates the different branches of the PTWs of (2), with U specified as the plant density variable, plotted as a function of the rainfall parameter $A$, by fixing the migration speed at $c=1.5$. The water diffusion coefficients for the panels are at $(a) d_{w}=0.01,(b) d_{w}=100,(c) d_{w}=300$ and $(d) d_{w}$ $=500$. The value of the other parameters are $d_{u}=1, B=0.45$, and $v=182.5$. Legend on the top of each panel shows the color of the locus of each solution branch. Note that the same line color is used for the $U_{\max }$ and $U_{\min }$. The light green line (middle line) in each panel indicates the $L 2$-norm of the solution of the traveling wave ODEs (4). The blue lines (upper line and lower line) exhibit the loci of maximum and minimum of the plant density variable $U$ respectively in each panel. The branches of solution begin from a Hopf bifurcation point marked by filled red square at $A=3.051,2.915,2.769$, 2.693 respectively for $d_{w}=0.01,100,300$, and 500. We observe that the values of the Hopf bifurcation points decrease gradually for the increasing values of $d_{w}=0.01,100,300$, and 500 respectively. There exists a fold point on the L2-norm marked by filled redcircle at $A=2.805,2.748$ respectively for $d_{w}$ $=300$, and 500. There also exist two fold points marked by filled red circles at $A=2.805,2.748$ on the locus of maximum and minimum of the plant density variable $U$ respectively for $d_{w}=300$, and 500 . There exists no fold point for $d_{w}=0.01$, and 100. We also observe that the $\mathrm{U}_{\max }$ increases as a function of $d_{w}$, e.g., for $A=1.0$, the $\mathrm{U}_{\max } \approx 16.3,18.4,22.75$, and 27.5 respectively when $d_{w}=$ $0.01,100,300$, and500. But, the $\mathrm{U}_{\min }$ remains almost constant for any value of $A$. 


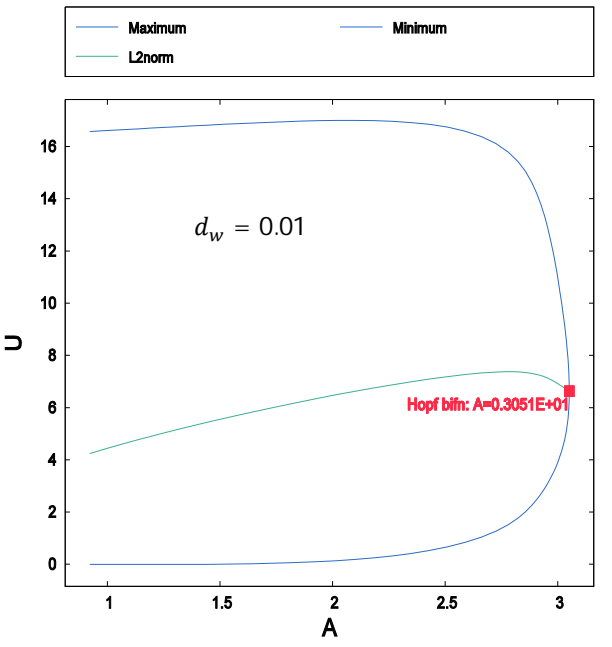

(a)

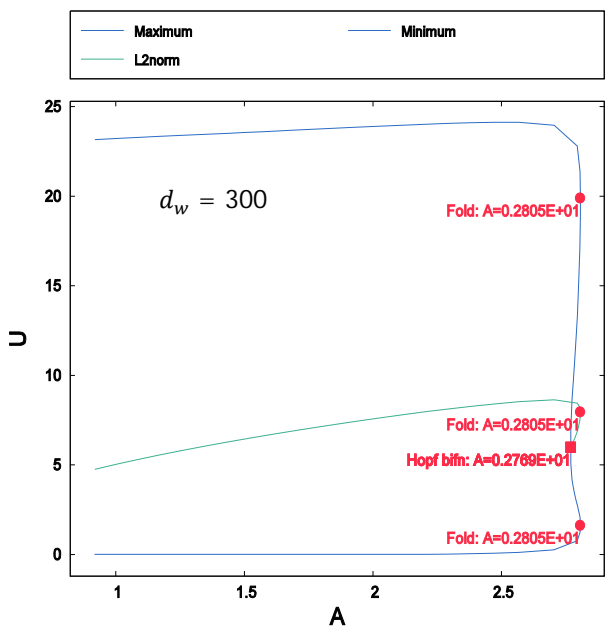

$(c)$

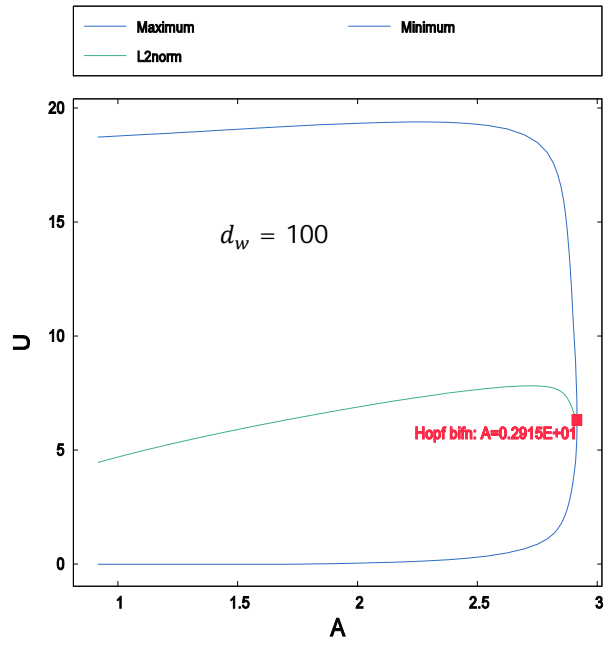

(b)

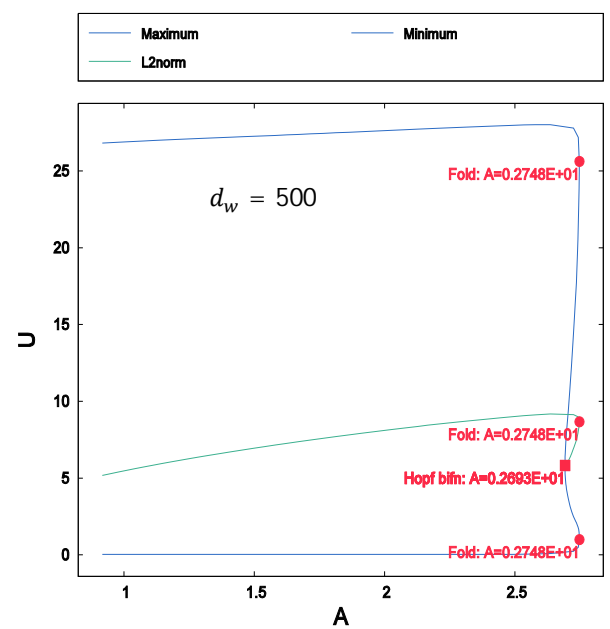

(d)

Fig. 8: The different bifurcation diagrams of the PTWs in the model (2),plotted $U$ as a function of the parameter $A$, for fixed migration speed $c=1.5$.

\subsection{Bifurcation diagrams of the PTWs: U versus $c$}

Figure 9 shows the different branches of the PTWs of (2), with U specified as the plant density variable, plotted as a function of the migration speed $c$, by fixing the rainfall parameter at $A=$ 2.5. The water diffusion coefficients for the panels are at $(a) d_{w}=0.01,(b) d_{w}=100,(c) d_{w}=$ 300 and $(d) d_{w}=500$. The values of the other parameter are $d_{u}=1, B=0.45$, and $v=182.5$. Legend on the top of each panel shows the color of the locus of each solution branch. The same line color is used for the $\mathrm{U}_{\max }$ and $\mathrm{U}_{\min }$. 


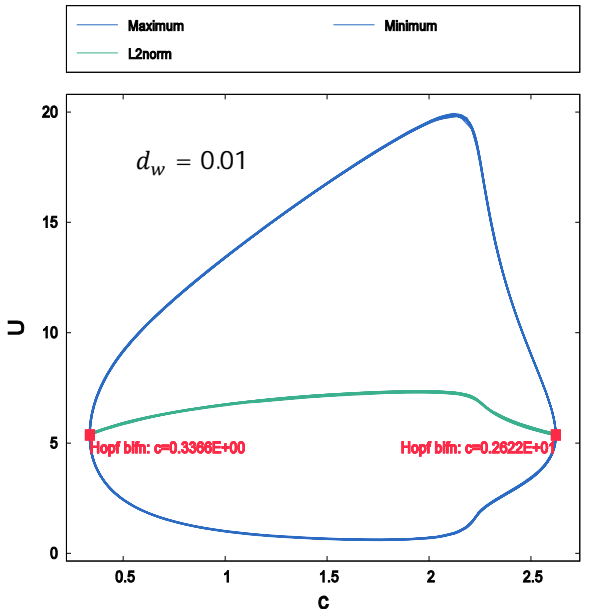

(a)

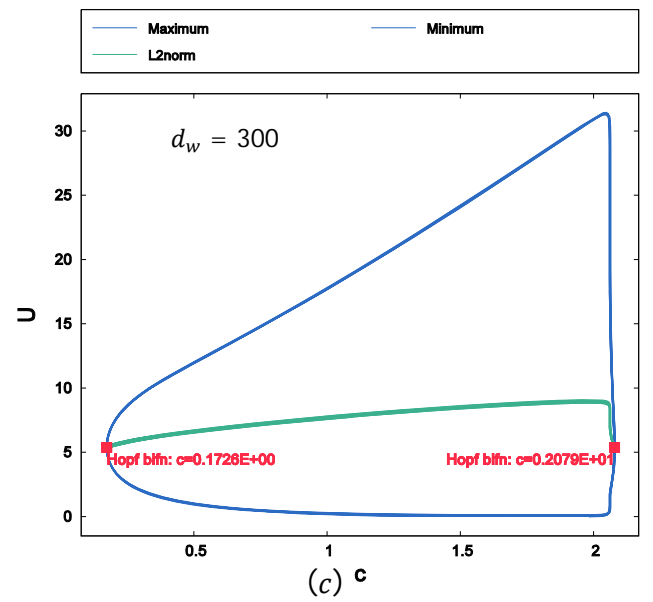

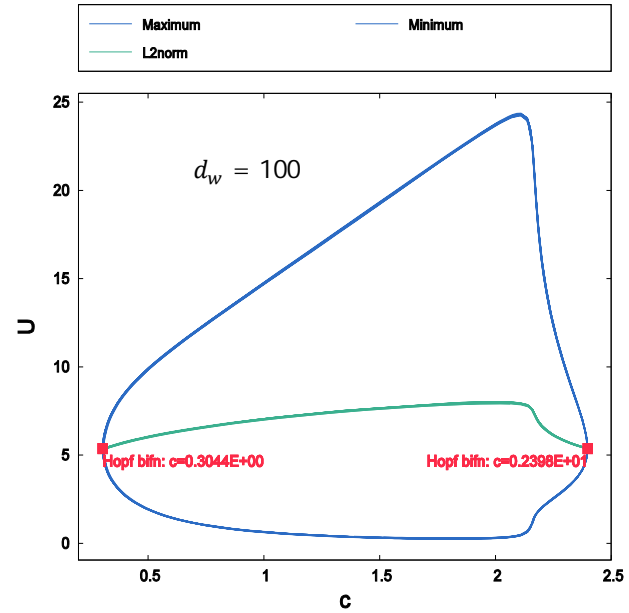

(b)
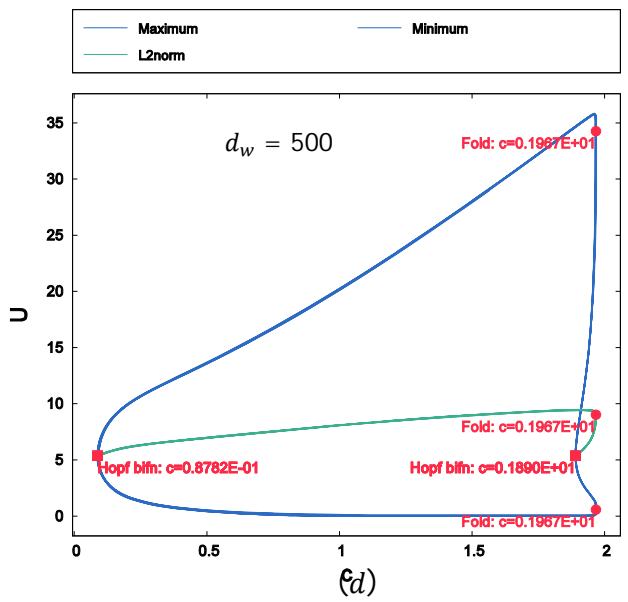

Fig. 9: The different bifurcation diagrams of the PTWs in the model (6.1),plotted $U$ as a function of the migration speed $c$, for fixed rainfall parameter $\mathrm{A}=2.5$.

The light green line (middle line) in each panelindicates the L2-norm of the solution of the traveling wave ODEs (4). The blue lines (upper line and lower line) exhibit the loci of maximum and minimum of the plant density variable $U$ respectively in each panel. The branches of solution emanate from a Hopf bifurcation point marked by filled red square at $c=0.3366,0.3044,0.1726$, 0.0878 respectively for $d_{w}=0.01,100,300$, and 500. Again, the branches of solution terminate to a Hopf bifurcation point at $c=2.622,2.398,2.079,1.890$ respectively for $d_{w}=0.01,100,300$, and 500. Therefore, the branches of PTWs are bounded with $c \in[0.3366,2.622]$ for $d_{w}=0.01, c \in$ [0.3044, 2.398] for $d_{w}=100, c \in[0.1726,2.079]$ for $d_{w}=300$ and $c \in[0.078,1.890]$ for $d_{w}=$ 500. It is to be mentioned that the ranges of the wave speed $c$ between the Hopf bifurcation points 
decrease gradually with the increase of water diffusion coefficient $d_{w}=0.01,100,300,500$ respectively. There exists a fold point or a saddle-node bifurcation point marked by filled red circle on the L2-norm at $c=1.967$ when $d_{w}=500$. There exist also two fold points marked by filled red circles at $c=1.967$ on the locus of the $U_{\max }$ and $U_{\min }$ for $d_{w}=500$. We observe that there exists no fold point for $d_{w}=0.01,100$, and 300 .

\section{Conclusion}

We have studied the existence of the periodic traveling wave solutions numerically, in the Klausmeier model and in the modified Klausmeier model. We have introduced an additional water diffusion term in (2b) to understand the behavior of the dynamics in order to understand the change of the existence of solutions as a function of water transport parameter, $d_{w}$. We have observed that, when the value of the parameter $A$ is increased, the corresponding period decreases (see Fig. 3(b)) and $L_{2}$-norm (see Fig. 3(a)) of the solution increases. We have briefly investigated the existence of the PTWs of the modified Klausmeier model (2) in the $A$ - $C$ parameter plane as a function of the water diffusion parameter. We have calculated a locus of Hopf bifurcation points and a locus of homoclinic solutions (PTWs having period infinity) in each panel in order to specify the right and left boundary respectively of the pattern forming region in the A-c parameter plane (see Fig.4 $(a-d)$ ).We have observed that the locus of homoclinic solutions and the locus of Hopf bifurcation points slightly change due to the increasing values of the water diffusion parameter $d_{w}=0.01,100,300$ and 500 respectively. The PTWs region gets shrink gradually for large wave speed as the water diffusion increases from panel (a) to panel (d) (see Fig. 4).We have established several types of bifurcation diagrams [39]for the models (1) and (2). Our results have showed a significant variation in migration speed. We have observed that, for a fixed rainfall, pattern wavelength is an increasing function of pattern migration speed $c$ (see Fig. 5). But this test is impossible due to insufficient field data on migration speeds. We have also observed that both $\mathrm{W}_{\max }$ and $\mathrm{U}_{\max }$ increase as a function of water diffusion coefficient $d_{w}$. On the other hand, $\mathrm{W}_{\min }$ and $\mathrm{U}_{\min }$ remain almost constant for any value of $A$ (see Fig. 6 and 8).In the Figure 7 and 9, we have mentioned that the ranges of the wave speed $c$ between the Hopf bifurcation points decrease gradually for the increasing values of water diffusion parameter. Therefore, it can be deduced that, the study of the existence of periodic traveling waves is essential to understand the complex spatiotemporal pattern formation in a reaction-diffusion-advection type of ecological model. However, in order to understand the detailed ecological complexity, one needs to study the stability of solutions thatwe areleaving for the publication in our forthcoming paper.

\section{BIBLIOGRAPHY}

[1] Macfadyen, W. A. (1950). Vegetation patterns in the semi-desert plains of British Somaliland. The Geographical Journal, 116(4/6), 199-211.

[2] Greig-Smith, P. (1979). Pattern in vegetation. The Journal of Ecology, 755-779.

[3] Lejeune, O., Couteron, P., \& Lefever, R. (1999). Short range co-operativity competing with long range inhibition explains vegetation patterns. Acta Oecologica, 20(3), 171-183. 
[4] Valentin, C., d'Herbès, J. M., \& Poesen, J. (1999). Soil and water components of banded vegetation patterns. Catena, 37(1), 1-24.

[5] Rietkerk, M., Dekker, S. C., de Ruiter, P. C., \& van de Koppel, J. (2004). Self-organized patchiness and catastrophic shifts in ecosystems. Science, 305(5692),1926-1929.

[6] Sherratt, J. A. (2005). An analysis of vegetation stripe formation in semi-arid landscapes. Journal of mathematical biology, 51(2), 183-197.

[7] Von Hardenberg, J., Meron, E., Shachak, M., \& Zarmi, Y. (2001). Diversity ofvegetation patterns and desertification. Physical Review Letters, 87(19), 198101.

[8] Adams, B., \& Carr, J. (2003). Spatial pattern formation in a model of vegetation-climate feedback. Nonlinearity, 16(4), 1339.

[9] Peters, D. P. C., \& Havstad, K. M. (2006). Nonlinear dynamics in arid and semi-arid systems: interactions among drivers and processes across scales. Journal ofArid Environments, 65(2), 196-206.

[10] Klausmeier, C. A. (1999). Regular and irregular patterns in semiarid vegetation.Science, 284(5421), 1826-1828.

[11] Borgogno, F., D'Odorico, P., Laio, F., \& Ridolfi, L. (2009). Mathematicalmodels of vegetation pattern formation in ecohydrology. Reviews of Geophysics, 47(1).

[12] Rietkerk, M., Boerlijst, M. C., van Langevelde, F., HilleRisLambers, R., de Koppel, J. V., Kumar, L., ... \& de Roos, A. M. (2002). Self-organization ofvegetation in arid ecosystems. The American Naturalist, 160(4), 524-530.

[13] Gilad, E., von Hardenberg, J., Provenzale, A., Shachak, M., \& Meron, E. (2007). A mathematical model of plants as ecosystem engineers, Journal of TheoreticalBiology 244 (4), 680-691.

[14] HilleRisLambers, R., Rietkerk, M., van den Bosch, F., Prins, H. H., \& de Kroon,H. (2001). Vegetation pattern formation in semi-arid grazing systems. Ecology, 82(1), 50-61.

[15] Ursino, N. (2007). Modeling banded vegetation patterns in semiarid regions:Interdependence between biomass growth rate and relevant hydrologicalprocesses. Water resources research, 43(4).

[16] Ursino, N., \& Contarini, S. (2006). Stability of banded vegetation patterns under seasonal rainfall and limited soil moisture storage capacity. Advances in WaterResources, 29(10), 1556-1564.

[17] Sherratt, J. A., \& Lord, G. J. (2007). Nonlinear dynamics and pattern bifurcations in a model for vegetation stripes in semi-arid environments. Theoretical population biology, 71(1), 1-11.

[18] Sherratt, J. A. (2010). Pattern solutions of the Klausmeier model for banded vegetation in semi-arid environments I. Nonlinearity, 23(10), 2657.

[19] Kealy, B. J., \& Wollkind, D. J. (2012). A nonlinear stability analysis of vegetative Turing pattern formation for an interaction-diffusion plant-surfacewater model system in an arid flat environment. Bulletin of Mathematical Biology, 74(4), 803-833.

[20] Van der Stelt, S., Doelman, A., Hek, G., \& Rademacher, J. D. (2013). Rise andfall of periodic patterns for a generalized Klausmeier-Gray-Scott model. Journalof Nonlinear Science, 23(1), 39-95.

[21] Salvucci, G. D. (2001). Estimating the moisture dependence of root zone waterloss using conditionally averaged precipitation. Water resources research, 37(5), 1357-1365.

[22] Callaway, R. M. (1995). Positive interactions among plants. The BotanicalReview, 61(4),306-349.

[23] Rietkerk, M., Ketner, P., Burger, J., Hoorens, B., \& Olff, H. (2000). Multiscale soil and vegetation patchiness along a gradient of herbivore impact in a semi-aridgrazing system in West Africa. Plant Ecology, 148(2), 207-224.

[24] Dunkerley, D. L., \& Brown, K. J. (2002). Oblique vegetation banding in theAustralian arid zone: implications for theories of pattern evolution andmaintenance. Journal of Arid Environments, 51(2), 163-181.

[25] Istanbulluoglu, E., \& Bras, R. L. (2006). On the dynamics of soil moisture,vegetation, and erosion: Implications of climate variability and change. Water Resources Research, 42(6). 
[26] Sherratt, J. A. (2015). Using wavelength and slope to infer the historical origin ofsemiarid vegetation bands. Proceedings of the National Academy of Sciences, 112(14), 4202-4207.

[27] Sherratt, J. A. (2016). When does colonisation of a semi-arid hillslope generatevegetation patterns?. Journal of Mathematical Biology, 73(1), 199-226.

[28] Siteur, K., Eppinga, M. B., Karssenberg, D., Baudena, M., Bierkens, M. F., \&Rietkerk, M. (2014). How will increases in rainfall intensity affect semiaridecosystems?. Water Resources Research, 50(7), 5980-6001.

[29] Sherratt, J. A. (2013). History-dependent patterns of whole ecosystems. Ecological Complexity, 14, 820 .

[30] Gilad, E., von Hardenberg, J., Provenzale, A., Shachak, M., \& Meron, E. (2004).Ecosystem engineers: from pattern formation to habitat creation. Physical Review Letters, 93(9), 098105.

[31] Ursino, N. (2005). The influence of soil properties on the formation of unstable vegetation patterns on hillsides of semiarid catchments. Advances in water resources, 28(9), 956-963.

[32] Edelstein-Keshet, L., Mathematical models in biology, Vol. 46, Siam, 1988.

[33] Sherratt, J. A. (2012). Numerical continuation methods for studying periodic travelling wave (wavetrain) solutions of partial differential equations. AppliedMathematics and Computation, 218(9), 4684-4694.

[34] Kopell, N., \& Howard, L. N. (1973). Plane wave solutions to reaction-diffusion equations. Studies in Applied Mathematics, 52(4), 291-328.

[35] Rademacher, J. D., Sandstede, B., \& Scheel, A. (2007). Computing absolute and essential spectra using continuation. Physica D: Nonlinear Phenomena, 229(2), 166-183.

[36] Gani, M. O., \& Ogawa, T. (2016). Stability of periodic traveling waves in the Aliev-Panfilov reaction-diffusion system. Communications in Nonlinear Science and Numerical Simulation, 33, 3042.

[37] Maginu, K. (1978). Stability of periodic travelling wave solutions of a nerve conduction equation. Journal of Mathematical Biology, 6(1), 49-57.

[38] Gani, M. O., \& Ogawa, T. (2015). Instability of periodic traveling wave solutions in a modified FitzHugh-Nagumo model for excitable media. Applied Mathematics and Computation, 256, 968-984.

[39] Gani, M. O., \& Ogawa, T. (2018). Spiral breakup in a RD system of cardiac excitation due to frontback interaction. Wave Motion, 79, 73-83. 